\title{
Association between etiology and lesion site in ischemic brainstem infarcts: a retrospective observational study
}

This article was published in the following Dove Press journal:

Neuropsychiatric Disease and Treatment

\author{
Gozde Baran' \\ Tugce Ozdemir Gultekin² \\ Oguz Baran ${ }^{3}$ \\ Cigdem Deniz ${ }^{3}$ \\ Salim Katar ${ }^{3}$ \\ Gulsen Babacan Yildiz ${ }^{2}$ \\ Talip Asil ${ }^{2}$ \\ 'Department of Neurology, Sisli \\ Hamidiye Etfal Research and \\ Training Hospital, ${ }^{2}$ Department of \\ Neurology, Bezmialem Vakif University, \\ ${ }^{3}$ Department of Neurosurgery, \\ Istanbul Research and Training \\ Hospital, Istanbul, Turkey
}

Background and purpose: To assess the anatomical distribution of the ischemic strokes of the brainstem, the effect of anatomical distribution on clinical features and prognosis, and the association between etiology and anatomical involvement.

Methods: A retrospective search of the patient database of our institution was performed for a total of 227 patients who were admitted to the Department of Neurology, Medical Faculty of Bezmialem Vakif University between January 2012 and September 2014. Patients with adequate diagnostic data and 3-month follow-up visit were included in the study.

Results: Twenty-one (9\%), $136(60 \%)$, and 65 (29\%) patients had an infarction only at the mesencephalon, pons, and medulla, respectively. However, a single patient $(0.5 \%)$ had an infarction both at the mesencephalon and pons, $3(1.5 \%)$ at the pons and medulla, and $1(0.5 \%)$ at the mesencephalon, pons, and medulla. While anterior involvement was more common in the mesencephalon and pons, posterior and lateral involvement occurred more frequently in the medulla. Large arterial atherothrombosis was the predominant cause of the strokes in all anatomical sites, particularly in infarcts involving the pons. Cardioembolic events were more common in patients with mesencephalic infarcts. Also, ischemia due to dissection was more common in infarctions involving the medulla, especially the lateral medulla. In subjects with simultaneous infarcts at other sites in addition to the brainstem, there was a significantly higher co-occurrence of medullary infarcts with cerebellar infarcts, mesencephalic infarcts with posterior cerebral artery infarcts, and pons infarcts with anterior circulation and multiple infarcts.

Conclusion: Determination of risk factors and infarct localization as well as prediction of etiological parameters may assist in improving survival rates and therapeutic approaches.

Keywords: cerebrovascular disorders, stroke, brain infarction, brain stem infarctions

\section{Introduction}

Strokes represent a major cause of morbidity and mortality worldwide, with ischemic strokes comprising $80 \%-90 \%$ of all strokes. Of the ischemic strokes, $25 \%$ involve the posterior circulation ischemic infarcts (POCI), and of these $60 \%$ and $40 \%$ occur in the brainstem and cerebellum, respectively. ${ }^{1,2}$ Brainstem infarctions develop as a result of posterior artery to artery embolism, cardioembolism, or involvement of the basilar artery (BA) and its branches due to hemodynamic causes, or manifest themselves as lacunar syndromes.

POCI occur at a lower frequency than infarcts involving the anterior circulation. It may be a challenging task to elucidate the association between etiology and infarct site due to different arterial supply to mesencephalon, pons, and medulla as well as due to the high frequency of normal variations, particularly in patients with brainstem
Sisli Hamidiye Etfal Research and Training Hospital, Neurology Clinic, Etfal Sk.,

3437 I Istanbul, Turkey

Tel +905325994462

Fax +90212 2240772

Email drgozdebaran@gmail.com 
involvement. There is relatively scarce published literature on the association between the etiology and the site of the infarction in the arterial territories of the brainstem. In contrast, a good body of evidence exists as to the anatomical sites involved in embolic and hemodynamic infarcts in the anterior circulation. A deeper insight into the underlying etiology may have utmost importance, particularly with regard to the establishment of secondary prevention strategies in patients experiencing ischemic stroke.

\section{Methods}

This study was undertaken at the Neurology Department, Medical Faculty of Bezmialem Vakif University, between January 2012 and September 2014, with the participation of 227 eligible patients out of 274 subjects with an established diagnosis of brainstem infarction among a total population of 1,752 cases admitted with an ischemic stroke. Data on patients were collected through a retrospective hospital database search. Demographic characteristics, laboratory and imaging study results, chronic conditions, risk factors, as well as neurological examination findings, NIHSS (National Institutes of Health Stroke Scale) scores, and MRS (Modified Rankin Score) at presentation, and third month MRS scores were recorded.

Only patients over 18 years of age with a brainstem infarction and/or accompanying anterior or posterior circulation infarcts who had a diffusion-weighted magnetic resonance imaging (DW-MRI) study within 7 days after presentation, in addition to imaging of the vascular tree using cranial magnetic resonance angiography (MRA) or computerized tomographic angiography (CTA), electrocardiography or transthoracic echocardiography (TTE), and when required, transesophageal echocardiography (TEE) and Holter monitoring, were included if they attended a follow-up visit at 3 months after stroke.

Exclusion criteria included the presence of conditions other than ischemic stroke (bleeding, tumor, etc.) as detected by cranial computed tomography (CT) or MRI, absence of the required etiological examinations, failure to attend the planned follow-up visits, failure of contact with the patient, presence of MR-incompatible metallic valvular or other types of prostheses, and diagnosis and follow-up based on CT only.

Medical assessment, neurological examination, routine blood tests, cranial imaging studies (CT +1.5 T MRI), cerebral angiography, 12-lead electrocardiogram (ECG), TTE/ TEE, and cardiac rhythm Holter monitoring were performed and recorded.

Patient characteristics recorded included the age, gender, presence of ischemic stroke, coronary artery disease, congestive heart failure, or cardiovascular risk factors, such as hypertension (HT, use of antihypertensive medications, a systolic and diastolic blood pressure of $\geq 140$ and $\geq 90 \mathrm{mmHg}$, respectively, recorded 2 weeks before or after stroke), diabetes (use of antidiabetic agents, a blood glucose measurement of $\geq 200 \mathrm{mg} / \mathrm{dL}$, or an $\mathrm{HbAlc} \geq 6.5 \%$ at presentation), or hyperlipidemia (use of antihyperlipidemic agents, or an low density lipoprotein (LDL) $\geq 130 \mathrm{mg} / \mathrm{dL}$ at presentation), and history of smoking.

Brainstem infarcts were classified into 3 main groups based on the site, as documented by DW-MRI: mesencephalon, pons, and medulla. Each of these anatomical locations were subdivided into 4 parts as follows: anteromedial $(\mathrm{AnM})$, anterolateral (AnL), lateral (L), and posterior (P), based on the classification system proposed by Burger et al. ${ }^{3}$ Furthermore, patients with the involvement of other anatomical sites (such as cerebellum, posterior cerebral artery [PCA], and anterior circulation) had data recorded. Patient groups were defined on the location of the infarct in 1 of the 3 sections of the brainstem (ie, mesencephalon, pons, and medulla) and/or in other locations. Patients with infarcts involving more than 1 site (AnM/AnL/L/Posterior) were entered in more than 1 group.

Furthermore, 5 patient subgroups were defined as follows on the basis of TOAST (trial of Org 10,172 in Acute Stroke Treatment) classification system for the etiology of the stroke: large arterial atherothrombosis, cardioembolism, small vessel disease, ischemic stroke due to other rare causes, and strokes of unknown cause. Intracranial and extracranial CTA or MRA was performed in all patients in order to visualize the vessel tree. Patients with $>50 \%$ narrowing in the vertebral and BA as documented by CTA/MRA were considered as those with large arterial atherothrombosis. A diagnosis of cardioembolic stroke was based on the presence of the following: atrial fibrillation (AF) in the ECG, paroxysmal AF in repeated ECG, akinetic segments or thrombus formation in TTE/TEE, history of rheumatoid valvular disease or cardiac valvular prosthesis implantation, paroxysmal AF in Holter monitoring, a recent history of myocardial infarction (MI) within the last 4 weeks, patent foramen ovale as shown by TEE or Bubble test, or presence of other low-risk cardioembolic causes despite absence of other main causes of stroke. A diagnosis of small vessel disease was suspected in infarcts with an MRI size of $<2 \mathrm{~cm}$ and associated with clinical manifestations such as the pure motor syndrome, pure sensory syndrome, sensory-motor syndrome, ataxic hemiparesis, and dysarthria-clumsy hand. However, if atherosclerotic narrowing was found in vascular imaging of these patients, then they were classified as having large arterial atherothrombosis. 
Rare etiological factors included a number of diagnoses such as vasculitis, arterial dissection, hypoperfusion syndromes, and malignancy. Patients with no detectable cause of stroke despite vascular imaging and cardiac risk assessment were classified as having stroke of unknown cause.

NIHSS was used to assess the severity of the stroke and was recorded at the time of initial presentation to the emergency room. An NIHSS of $<6$ was considered mild, and NIHSS $\geq 6$ was considered severe stroke.

Dependency and functional improvements were assessed using MRS, where scores of 1 and 2 , or $\geq 3$ were considered to be independent, and dependent of others, respectively. Also, MRS scores of 0,1 , and 2 were considered to indicate good prognosis, while scores of $3,4,5$, and 6 were considered to signify poor prognosis. MRS scores at presentation and at 3 months after stroke were determined to assess the rate of improvement. The time and cause of deaths were recorded. Recurrent strokes occurring within 3 months of the initial event were also recorded.

The study protocol was approved by the Ethics Committee, Bezmialem Vakif University. It was not considered necessary to get consent from patients by the ethics Committee of Bezmialem Vakif University because the study was a retrospective data analysis. We certify that confidentiality of patient data was respected.

\section{Statistical analysis}

SPSS 16 for Windows statistical software pack was used for statistical analysis. All parameters were presented as mean \pm SD. Data comparison was performed using the chi-square, Fisher's exact chi-square, Wilcoxon tests, and binary logistic regression analysis. The significance of the results was assessed using the $P$ level. A $P$ level of $<0.05$ was considered to signify statistical significance.

\section{Results}

The data obtained from 227 patients with documented brainstem infarcts were examined. Of the overall population, $136(60 \%)$ were male and $91(40 \%)$ were female.

Large arterial atherothrombosis, cardioembolism, small vessel disease, and other rare causes of the stroke were determined in 129 (57\%), 51 (22.5\%), 15 (6.5\%), and $15(6.5 \%)$ of the patients, respectively. A total of 17 patients $(7.5 \%)$ were classified as having a stroke of unknown cause after a failure to determine the etiological cause despite a comprehensive assessment. In the group with stroke due to other rare causes, there were 7 cases with vertebral artery (VA) and/or BA dissection, 6 cases with vasculitis, and 2 patients with malignancy.
Chronic conditions that could be associated with an underlying atherosclerotic process included HT in 174 (76.5), while there were 113 (50\%) diabetics (DM), 77 (34\%) hyperlipidemics, $14(6 \%)$ with congestive heart failure (CHF), $51(22.5 \%)$ with coronary artery disease, and 58 (25.5\%) with a previous history of stroke, while 101 patients $(44.5 \%)$ had a history of chronic tobacco use at some time in their life.

With regard to an etiological assessment of the cardioembolic strokes, 27 patients $(11.5 \%)$ were found to have AF in ECG, while 18 (7.5\%) had paroxysmal atrial fibrillation in Holter monitoring, and 45 (20\%) individuals were found to have a cardioembolic etiology in TTE/TEE.

Other comorbidities included vasculitis in 6 patients (2.5\%; 2 with Behcet's disease), malignancy in 5 (2\%; lung cancer, colon cancer, breast cancer, nasopharynx cancer, and non-Hodgkin lymphoma; 1 each), renal transplant in 1, and familial Mediterranean fever in 2 patients.

Stroke site in the brainstem included only a mesencephalon infarct in $21(9 \%)$, pons infarct in $136(60 \%)$, and medulla infarct in 65 (28.5\%). Also, simultaneous infarcts in mesencephalon and pons, pons and medulla, and mesencephalon, pons, and medulla were detected in $1(0.5 \%), 3(1.5 \%)$, and $1(0.5 \%)$ patients, respectively (Table 1$)$.

There were a total of 24 mesencephalon infarcts in the study population, which involved AnM, AnL, posterior, and $\mathrm{L}$ regions in 10 (41.5\%), 7 (29\%), 6 (25\%), and 1 (4\%) patients, respectively.

There were 164 infarcts in pons, which involved AnM, AnL, posterior, and L regions in 50 (30.5\%), 53 (32.5\%), 23 (14\%), and 38 (23\%) patients, respectively.

The corresponding figures for 79 medulla infarcts were $15(19 \%), 9(11.5 \%), 19(24 \%)$, and $36(45.5 \%)$, respectively (Table 1).

From an anatomical viewpoint, the most commonly involved sites included AnM in mesencephalon, AnM and AmL in pons, and $\mathrm{L}$ in medulla. An overall assessment of the brainstem infarcts showed that the anterior pons was the most commonly involved site, while the lateral mesencephalon was the least frequently involved site.

Fifty-one (22.5\%) of the 227 participants had simultaneous non-brainstem infarcts in addition to brainstem infarcts: $23(10 \%)$ in cerebellum, $9(4 \%)$ in PCA territory, $8(3.5 \%)$ in the anterior circulation, and $11(5 \%)$ in multiple areas (cerebellum + PCA in 8 , cerebellum + anterior circulation in $2, \mathrm{PCA}+$ anterior in 1 ).

When patients having infarcts in addition to those detected in the brainstem were examined using the Fisher-FreemannHalton test, patients with additional cerebellar infarcts were significantly more likely to have infarcts in the medulla of 
Table I Lesion sites: distribution of lesions

\begin{tabular}{llllll}
\hline & Total $^{\mathbf{a}}$ & Anteromedial (\%) & Anterolateral (\%) & Lateral (\%) & Posterior (\%) \\
\hline Mesencephalon & 24 & $10(41.5)$ & $7(29)$ & $1(4)$ & $6(25)$ \\
Pons & 164 & $50(30.5)$ & $53(32.5)$ & $38(23)$ & $23(14)$ \\
Medulla & 79 & $15(19)$ & $9(11.5)$ & $36(45.5)$ & $19(24)$ \\
\hline
\end{tabular}

Notes: ${ }^{\text {TT }}$ wo of the patients with mesencephalon infarcts, 5 of the patients with pontine infarcts, and 4 of the patients with medullary infarcts had an infarct in other locations of the brainstem as well (Mes+P:I, P+med:3, Mes+P+med:I). Also, there were infarcts in other localizations of the brainstem in I of the patients with mesencephalon infarct, 7 of the patients with pontine infarct, and 3 of the patients with medullary infarct (anteromedial/anterolateral/lateral/posterior infarct).

Abbreviations: Mes, mesencephalon; P, pons; med, medulla.

the brainstem, patients with additional PCA infarcts were significantly more likely to have infarcts in mesencephalon of the brainstem, and those with additional multiple infarcts involving the posterior circulation were significantly more likely to have a brainstem infarct in pons $(P<0.001)$.

The 3 anatomical regions of the brainstem were also examined with respect to TOAST etiological classification system (Table 2).

Accordingly, of the 23 patients with mesencephalon infarct, 21 had infarcts only in the mesencephalon, while 1 patient had an infarct in mesencephalon + pons, and 1 in mesencephalon + pons + medulla. A large artery atherothrombosis was the etiological factor in 11 (48\%) of the 23 patients, while a cardioembolic cause was found in $6(26 \%)$, and small vessel occlusion in $2(8.5 \%) ; 1$ patient $(4.5 \%)$ had a stroke due to a rare etiological condition that could be detected. The cause of the stroke could not be determined in 3 patients (13\%) despite investigations.

Pons was the only site involved in 136 of the 141 patients with pontine infarction, while 1 had co-existent infarcts in mesencephalon and pons, 3 had infarcts in pons and medulla, and 1 had simultaneous infarcts in all 3 regions. The distribution of patients with pons infarcts according to TOAST classification showed a stroke occurring due to larger arterial atherothrombosis, cardioembolic disease, or small vessel occlusion in $86(74.0 \%), 30(21 \%)$, and $11(8 \%)$ cases, respectively, while $6(4 \%)$ had infarcts due to other rare causes, and $8(5.5 \%)$ had no detectable cause of stroke, despite a search for it (Table 2).

Sixty-nine of the 227 patients had a medulla infarction. Of these, the infarct was only at the medulla in 65, while in both pons and medulla in 3, and in mesencephalon, pons, and medulla in 1. TOAST classification in patients with medulla infarcts showed large artery atherothrombosis, cardioembolism, small vessel occlusion, and other rare etiologies in $37(53.5 \%), 15(21.5 \%), 2(3 \%)$, and $8(11.5 \%)$ of the cases, respectively. The etiology of the stroke could not be determined despite investigations in $7(10 \%)$ of the cases with medulla infarction (Table 2).

In another classification assessing the association between the site of the brainstem infarcts and etiological causes, the brainstem was divided into the following 4 regions: AnM, AnL, L, and P. With regard to this classification, 75, 71, 73, and 49 of the 227 patients were found to have AnM, AnL, $\mathrm{L}$, and posterior infarcts, respectively.

Also the distribution of these 4 regions (ie, AnM, AnL L, and posterior) according to the TOAST classification was evaluated. Of the 75 patients with AnM infarcts, 41 (54.5\%), $17(22.5 \%), 5(6.5 \%)$, and $3(4 \%)$ were found to have a stroke associated with large artery atherothrombosis, cardioembolic causes, small vessel occlusion, and other rare causes of stroke. No cause could be determined in 9 patients $(12 \%)$ despite etiological investigations (Table 3 ).

Among 71 patients with AnL infarctions, TOAST classification showed the presence of stroke associated with large artery atherothrombosis, cardioembolism, small vessel occlusion, and other rare cerebrovascular conditions in $43(57.5 \%), 15(21 \%), 6(8.5 \%)$, and $2(3 \%)$ of the patients, respectively. No etiology could be detected in 5 patients $(7 \%)$ despite investigations (Table 3).

The TOAST classification distribution of 73 patients with lateral infarcts showed that of these patients, 43 (59\%), 18 $(24.5 \%), 2(2.5 \%)$, and $7(9.5 \%)$ had an infarct due to large artery thrombosis, cardioembolic causes, small vessel occlusion,

Table 2 Etiological distribution of brainstem infarcts according to the anatomical site

\begin{tabular}{lllllll}
\hline & Total $^{\text {a }}$ & $\begin{array}{l}\text { Large artery } \\
\text { atherothrombosis (\%) }\end{array}$ & Cardioembolic (\%) & $\begin{array}{l}\text { Small vessel } \\
\text { occlusion (\%) }\end{array}$ & $\begin{array}{l}\text { Other rare } \\
\text { causes (\%) }\end{array}$ & $\begin{array}{l}\text { Unknown } \\
\text { cause (\%) }\end{array}$ \\
\hline Mesencephalon & 23 & $1 \mathrm{I}(48)$ & $6(26)$ & $2(8.5)$ & $1(4.5)$ & $3(13)$ \\
Pons & 141 & $86(74)$ & $30(2 I)$ & $11(8)$ & $6(4)$ & $8(5.5)$ \\
Medulla & 69 & $37(53.5)$ & $15(21.5)$ & $2(3)$ & $8(11.5)$ & $7(10)$ \\
\hline
\end{tabular}

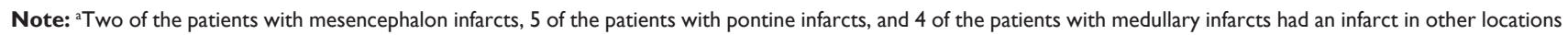
of the brainstem as well (Mes+P:I, P+med:3, Mes+P+med:I).

Abbreviations: Mes, mesencephalon; $P$, pons; med, medulla. 
Table 3 Etiological distribution

\begin{tabular}{lllllll}
\hline & Total & $\begin{array}{l}\text { Large artery } \\
\text { atherothrombosis (\%) }\end{array}$ & Cardioembolic (\%) & $\begin{array}{l}\text { Small vessel } \\
\text { occlusion (\%) }\end{array}$ & $\begin{array}{l}\text { Other rare } \\
\text { causes (\%) }\end{array}$ & $\begin{array}{l}\text { Unknown } \\
\text { cause (\%) }\end{array}$ \\
\hline Anteromedial & 75 & $4 I(54.5)$ & $17(22.5)$ & $5(6.5)$ & $3(4)$ & $9(12)$ \\
Anterolateral & 71 & $43(57.5)$ & $15(2 I)$ & $6(8.5)$ & $2(3)$ & $5(7)$ \\
Lateral & 73 & $43(59)$ & $18(24.5)$ & $2(2.5)$ & $7(9.5)$ & $3(4)$ \\
Posterior & 49 & $31(63)$ & $6(12)$ & $3(6)$ & $3(6)$ & $6(12)$ \\
\hline
\end{tabular}

Note: Patients with infarcts at multiple sites were counted in each group separately.

and other rare causes of stroke, respectively. No etiology could be found in 3 (4\%) despite investigations (Table 3).

The corresponding figures for TOAST classification among 49 patients with posterior infarcts were 31 (63\%), $6(12 \%), 3(6 \%)$, and $3(6 \%)$. Etiology was unclear despite a diagnostic work-up in 6 cases (12\%) (Table 3).

Infarcts were also detected in areas other than brainstem in 51 patients who were included in the study. TOAST classification stroke etiologies of these 51 patients showed that $25(49 \%)$ patients had large artery atherothrombosis, $16(31.3 \%)$ patients had cardioembolism, 1 (1.9\%) had minor vascular disease, $6(11.7 \%)$ were due to other rare causes, and in $3(5.8 \%)$, the cause could not be determined even though the necessary tests were performed. Although the large artery atherothrombosis was again the most common cause of strokes, patients with co-existent non-brainstem strokes were significantly more likely to have stroke of cardioembolic origin. On the other hand, in those with small vessel disease, stroke was rarely associated with multiple infarcts. The statistical assessment based on the chi-square test showed no significant differences in terms of TOAST etiology between those who had a brainstem infarction only and those who had simultaneous infarcts at sites in addition to the brainstem infarct $(P=0.097)$.

In order to determine the level of dependency in carrying out daily activities and the rate of improvement, MRS scores at presentation and 3 months thereafter were calculated. Accordingly, the mean MRS score was $3.27 \pm 1.06$ at presentation versus $1.99 \pm 1.61$ at 3 months. In 124 of the patients $(54.5 \%)$, the MRS score at presentation was $\leq 2$, and it was $\geq 3$ in the remaining 103 patients (45.5\%). At 3 months after presentation, the number of patients with an MRS score of $\leq 2$ and $\geq 3$ was $152(67 \%)$ and 75 (33\%), respectively. Of these 75 patients, $6(2.5 \%)$ could not be followed in the first 3-month period due to death.

NIHSS was used to assess the severity of stroke. The average NIHSS at presentation in 227 patients was $6.37 \pm 4.21$ (min: 0, max: 24). The neurological examination score was $\leq 5$ and $\geq 6$ in 112 (49.3\%; mean: 3.38, SD: 1.25) and 115 (mean: 9.3, SD: 4.04), respectively, of the patients at presentation.

\section{Discussion}

Determination of the cause of and risk factors associated with the development of stroke carries significance with regard to administration of appropriate management and secondary prevention strategies. More widespread availability and use of diagnostic radiological and laboratory methods allow the clinicians to determine the etiology of stroke in a higher proportion of patients than before. Also, the anatomical site of the infarction may provide clues with regard to the etiology of the stroke.

Ischemic vertebrobasilar strokes comprise nearly $23 \%$ of all ischemic strokes of the brain, while ischemic brainstem infarcts are responsible for $11 \%$ of all ischemic strokes. ${ }^{4,5}$ Of the ischemic vertebrobasilar infarcts, $27 \%, 14 \%$, and $7 \%$ occur in pons, medulla, and mesencephalon. Cerebellar involvement is observed in $7 \%$ of the patients, while PCA territory is involved in $36 \%$, and multiple sites in $9 \%{ }^{4,6}$

In a 2014 study by Lin et al, of the 199 patients with brainstem infarcts, $170(85.4 \%)$ were found to have pons infarcts, while $20(10.1 \%)$ had medulla infarcts and $3(1.5 \%)$ had mesencephalon infarcts. In the remaining 6 patients (3.0\%), the infarct also occurred in non-brainstem sites. ${ }^{7}$

In $141(62 \%), 69(30.5 \%)$, and $23(10 \%)$ of our 227 participants, there was an infarct involving pons, medulla, and mesencephalon, respectively, consistent with previous reports.

According to the TOAST classification system, large artery atherothrombosis comprises the largest etiological group in strokes. A significant cause of strokes involving the posterior circulation is represented by the atherosclerotic plaques in the aortic arch. ${ }^{8,9}$ When the thrombus is formed within the extracranial VA, the clot formation usually takes place at the point of atherosclerotic narrowing. ${ }^{10,11}$ On the other hand, clots forming within the intracranial VA usually extend over to the proximal BA. ${ }^{10}$ The distal part of the BA consists of the proximal PCAs and atherosclerotic lesion sites. ${ }^{10,12}$ A thrombus within BA generally may reach the orifice of the other long circumferential cerebellar arteries (anterior inferior cerebellar artery [AICA]) or superior cerebellar artery (SCA). The embolism frequently involves 
the distal branches of BA and particularly tends to clog the branches of the posterior inferior cerebellar artery (PICA), or less frequently, the proximal VA or BA. ${ }^{13}$ Atherosclerosis may also affect the branches of VA as well as BA. ${ }^{14}$ AICA arises from BA, and AICA occlusions are of atherothrombotic nature in many patients. ${ }^{15-17}$ Conversely, atherothrombosis and cardioembolism play an equal role in the development of PICA occlusions, while cardioembolism is more frequent in SCA occlusions. Small penetrating branches may also be affected by the occlusive disease. ${ }^{18}$

In the 2014 study by Lin et al involving 199 patients with brainstem infarcts, stroke was due to small vessel occlusion, larger artery atherothrombosis, undetermined causes, or cardioembolism in 77 (38.7\%), 74 (37.2), 25 (12.6\%), and $23(11.6 \%)$ of the individuals. These authors did not report any patients who had stroke due to other rare causes. ${ }^{7}$ In our study, the most frequent etiological cause was large arterial atherothrombosis, followed by cardioembolism, while small vessel disease, other rare causes, and undetermined causes played a less significant etiological role. A subclassification of our patients based on both the 3 anatomical regions (mesencephalon/pons/medulla) and the vascular territories (AnM, AnL, L, posterior) also showed that the most frequent etiology was the large artery atherothrombosis in all groups. Among strokes occurring in mesencephalon, pons, or medulla, the highest frequency of large artery atherothrombosis was observed in pons, ie, $74 \%$ (Table 2 ). An examination of the etiology of the large artery atherothrombosis according to the arterial territories demonstrated that large artery atherothrombosis was responsible for $>50 \%$ of the strokes in all 4 groups and represents the most frequent etiological factor (Table 3).

Another etiological factor in the development of strokes includes cardioembolism, which comprises $15 \%-30 \%$ of all strokes. ${ }^{19,20}$ In lesions involving both hemispheres in the anterior or posterior circulation and bilateral or multiple posterior circulatory infarctions, cardioembolism should be suspected. ${ }^{21}$ Examination of the etiology in our patient group showed that in mesencephalon, which is supplied by SCA, cardioembolic etiologies occurred at a higher frequency, that is, $26 \%$, as compared with other localizations. The observation that 16 of the 51 patients $(31.5 \%)$ with simultaneous infarcts in non-brainstem locations had cardioembolic stroke is supportive of the notion that this etiological factor is also more significantly increased in patients with multiple infarcts. However, neither in patients with mesencephalon infarcts nor in patients with simultaneous brainstem and non-brainstem infarcts, no significant increase in the frequency of cardioembolic etiology was observed $(P>0.05)$.
According to the TOAST classification, small artery atherothrombosis represents another cause of strokes. In our study, 15 of the 227 patients $(6.5 \%)$ had small vessel disease. With respect to arterial territories, the frequency of small vessel disease was lower in lateral infarcts than in other locations (Table 3). Also, the frequency of infarcts due to small vessel occlusion was lower in our study than in previous reports. It is plausible that atherosclerotic strokes were more likely to be detected in our study, since vessel imaging was performed in all of our patients, despite initial suggestion of small vessel disease in MR images with respect to lesion site and clinical manifestations.

The 4th etiological group in TOAST classification consists of the rare causes of stroke, such as dissections, vasculitic disorders, and malignancy. Seven of 15 patients (3\%) with other rare causes of stroke in our study had dissection, 6 in VA and 1 in BA. Lateral medulla and cerebellum represent the sites with highest susceptibility to ischemia induced by extracranial VA dissection. ${ }^{22-24}$ Arterial dissections, however, cause pons strokes rarely. ${ }^{25,26}$ Of all the mesencephalic strokes, $4.8 \%$ are caused by arterial dissections. ${ }^{27}$ Of the 7 patients in our study with dissection, 2 had an infarct in pons ( 1 in pons AnM, the other in pons $\mathrm{AnL}$ ), and the remaining patients had an infarct in medulla ( 1 in AnM, 2 in AnL, and 2 in L). Also 2 patients had additional cerebellar infarction.

Vasculitic disorders, classified in the rare causes of stroke, are a heterogeneous group of diseases characterized by the inflammation and necrosis of the vessel wall. One such condition associated with vasculitis, Behcet's disease, is known to mainly involve the brainstem. ${ }^{28}$ Six patients $(2.5 \%)$ in our group had vasculitis, and 2 were found to have Behcet's disease. In both of these patients, infarction was located anteriorly in the pons, and these cases had a previous history of stroke. Of these 6 patients with vasculitisassociated stroke, 4 and 2 had pons and medulla infarcts, respectively. Also, 4 of these patients had lateral involvement in the brainstem.

According to TOAST classification, malignancies also represent another rare cause of stroke. Approximately 15\% of the patients with malignancy endure a thromboembolic event during the course of their disease. ${ }^{29}$ In our study, of the 15 cases with rare causes of stroke, 5 (2\%) had malignancy. However, in 2 of these patients, vessel imaging showed $>50 \%$ narrowing in VA consistent with larger artery atherothrombosis, thus these patients were classified in the group of large artery atherothrombosis. Also, another patient had multiple ischemic lesions consistent with small vessel disease in the anterior and posterior systems, and therefore this patient was categorized as having small vessel 
disease. There were 2 patients in whom malignancy was thought to be the cause of stroke, and 1 of these cases had a stroke in posterior mesencephalon and the other in the lateral medulla.

Despite comprehensive diagnostic work-up, no cause for stroke could be determined in 17 patients $(7.5 \%)$. The proportion of strokes with an undetermined cause in mesencephalon, pons, and medulla was $13 \%, 5.5 \%$, and $10 \%$, respectively (Table 2). Also, no cause could be determined in $12 \%, 7 \%, 4 \%$, and $12 \%$ of the infarcts in $A n M, A n L, L$, and posterior sites, respectively.

Isolated mesencephalon infarcts are rare, and patients generally have involvement in other sites such as cerebellum, thalamus, and pons. Ischemic mesencephalic infarcts result from large arterial disease (39\%), small artery disease (13\%-24\%), cardiogenic and artery-to-artery embolism $(20 \%-46 \%)$, in situ thrombosis $(2 \%-23 \%)$, arterial dissection $(0 \%-5 \%)$, and unknown causes $(10 \%) .{ }^{27}$ In a 2005 study by Kim and $\mathrm{Kim}^{44}$ involving 37 patients with isolated mesencephalon infarction, 18 had an infarct at AnM, while 11, 6, and 2 had an infarct at AnL, AnM + AnL, and L, respectively. Of the 18 patients with infarcts at $A n M, 10$ had small vessel disease and 8 had large artery atherothrombosis. Of the 11 AnL infarcts, 9 (82\%) had large arterial atherothrombosis. In 5 of 6 patients with AnM plus AnL infarcts, large artery atherothrombosis was the etiological factor. The authors concluded that while large artery atherothrombosis and small vessel occlusion had a more marked role in the pathogenesis, cardioembolic causes were less frequent. In our study, the leading etiological factor among 23 patients with mesencephalon infarcts was the large artery atherothrombosis that was found in $11(47.8 \%)$ of these subjects. Etiological classification based on arterial territories showed large artery atherothrombosis in 5 of 7 patients with mesencephalon AnL infarction. In other sites, no association could be observed with etiological classification.

Isolated pons infarcts comprise $3 \%$ of all ischemic strokes. ${ }^{30}$ Ischemic pontine strokes mostly develop due to the stenosis or occlusion of BA, and particularly atherosclerosis of the BA branches (39\%), as well as due to small vessel disease $(21 \%)$, and larger arterial (vertebrobasilar) disease $(18 \%) .{ }^{31}$ Of all pontine ischemic strokes, only $8 \%$ are due to cardioembolism, since emboli generally lodge more distally in the pons. ${ }^{30}$ No causative factors can be determined in 11 of the pontine infarcts. In a 150 patient study by Kumral et al examining the etiological factors in pontine arteries, $59(39 \%)$, $31(21 \%), 27(18 \%)$, and $12(8 \%)$ were found to have BA branch disease, small vessel occlusions, atherothrombosis of the vertebrobasilar arteries, and cardioembolism, respectively, while the cause of the stroke could not be determined in 16 individuals (11\%). These results show that BA branch disease and small vessel disease comprise the most frequent etiological factors in pontine infarcts. ${ }^{30}$ Again, Vemmos et al investigated the etiology of the isolated pontine infarcts in 100 patients. In that study, BA branch disease, small arterial occlusions, and large artery atherothrombosis were the cause of the infarct in $43 \%, 34 \%$, and $21 \%$ of the cases, respectively. ${ }^{32}$ In the current study, the major cause of pontine infarction among 141 patients was the large artery atherothrombosis that occurred in 86 cases (74.0\%).

Infarctions involving the medulla are rare, with lateral medullar infarcts occurring almost 5 times more frequently than medial infarcts. ${ }^{25} \mathrm{Kim}$ et al found isolated medulla infarcts in $87(61.2 \%)$ of 142 patients with acute ischemic stroke, while 55 patients had infarcts in sites other than medulla. Of these 55 patients, 43 were found to have cerebellar infarction. In all patients with cerebellar infarcts, PICA territory was involved. Ninety-three patients had lateral infarcts, and 39 had infarcts at AnM and 22 had posterior infarcts. In that study, only 2 patients had concomitant medial (AnM and AnL) medulla infarcts with lateral or posterior medulla lesions. ${ }^{33}$ In our study, 43 of the 69 patients $(62.5 \%)$ with medullary infarction had an isolated medullary infarct, while 13 had concomitant cerebellar and 1 had concomitant anterior circulation infarcts, and 2 were found to have infarcts at multiple sites. Among patients with concomitant brainstem and cerebellar infarcts, medullary infarcts were significantly more common $(P<0.001$; Table 4$)$.

The most frequent cause of medullary infarcts is the atherosclerosis of VA or its branches. ${ }^{34}$ Lateral medullary infarcts generally result from the large artery atherothrombosis of VA (67\%) or PICA (10\%). Another important cause of lateral and medial medullary infarcts is VA dissection $(20 \%-30 \%) .{ }^{25}$ In the study by Kim et al, a differential etiology depending on the vascular territory has been reported for the medullary infarcts. Accordingly, the most frequent cause was arterial atherothrombosis (34.5\%), while lacunar and cardioembolic strokes were more uncommon $(3.5 \%$ and $4.2 \%$, respectively). Also, other causes of stroke comprised $10.6 \%$ of the cases $(9.2 \%$ VA dissection, $1.4 \%$ anti-phospholipid antibody syndrome).$^{33}$ In their study, cardioembolism and other identified causes of stroke occurred in lateral regions supplied by PICA or VA only, or in the posterior region supplied by the posterior spinal artery, while lacunar infarcts occurred in only 5 patients who had anteriomedial medullary infarction. ${ }^{33,35}$ The observation by Shono et al that small vessel disease was the most frequent cause of medial medullary infarcts is supportive of this finding. ${ }^{36}$ 
Table 4 Distribution of the anatomical site of non-brainstem infarcts

\begin{tabular}{lllll}
\hline & Mesencephalon & Pons & Medulla & $\begin{array}{c}\text { Mesencephalon }+ \\
\text { pons }\end{array}$ \\
\hline Cerebellum & 2 & 7 & 13 & I \\
PCA & 7 & 2 & 0 & 0 \\
Anterior circulation & $\mathrm{I}$ & 6 & $\mathrm{I}$ & 0 \\
Multiple $^{\mathrm{a}}$ & $\mathrm{I}$ & 8 & 2 & 0 \\
Total & $\mathrm{II}$ & 23 & $\mathrm{I}$ & $\mathrm{I}$ \\
\hline
\end{tabular}

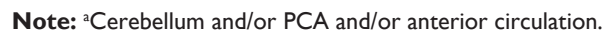

Abbreviation: PCA, posterior cerebral artery.

In our study, the distribution of patients with medullary infarcts according to TOAST classification showed that large arterial atherothrombosis was the most frequent cause with 37 patients $(53.5 \%)$, while 15 patients $(21.5 \%)$ were thought to have stroke of cardioembolic causes. Of the medullary infarcts due to cardioembolic stroke, 5 were in medulla AnM, 2 were in medulla AnL, 5 were in medulla $\mathrm{L}$, and 2 were in posterior medulla. Also a single patient had concurrent infarction in lateral and posterior regions. Two patients (3\%) had stroke due to small vessel occlusion, while 8 cases $(11.5 \%)$ were found to have other causes of stroke. In the remaining 5 patients with rare causes of stroke, dissection was detected. In these patients, infarcts were located at AnM, L, and posteriorly in the medulla in 1, 2, and 2 of the patients, respectively. In the overall patient group, the observation that 5 of the 7 dissections were located at the medulla is supportive of the view that dissection-induced brainstem infarcts occur more commonly in the medulla. Additionally, in 7 patients (10\%) with medullary infarcts, the etiology of the stroke could not be determined despite investigations (Table 2).

When the brainstem is considered as a whole, $75,71,73$, and 49 patients had an infarct located in AnM, AnL, L, and posteriorly, respectively (Table 3). While AnM, AnL, and lateral territories were involved at a similar frequency, posterior infarcts were less common. The most common etiological factor in all arterial territories was the large artery atherothrombosis, followed by strokes of cardioembolic origin.

Cerebellar blood supply is provided by SCA, AICA, and PICA. SCA infarcts comprise the most common cerebellar stroke syndrome. ${ }^{37-42}$ AICA supplies the lateral lower pons and lateral upper medulla, ${ }^{16}$ while PICA mostly causes lateral medullarly infarcts in the brainstem. ${ }^{42,43}$ In our study, among the 51 patients with simultaneous infarcts at other locations in addition to those at the brainstem, 23 had only cerebellar infarcts in addition to brainstem infarcts. In those with concurrent cerebellar infarcts, medullary brainstem infarcts were significantly more common $(P<0.05)$.
An assessment of the lesion site in relation to anatomical regions showed that AnM was the most commonly involved site in mesencephalon, while AnM and AnL were most commonly involved in the pons, and L in the medulla. In the overall assessment, lateral mesencephalon was the least frequently involved site among all the anatomical sites. When mesencephalon, pons, and medulla were considered together in the brainstem, AnM, AnL, and L involvement occurred at a similar frequency, with the least involvement frequency posteriorly.

Large artery atherothrombosis represented the most common etiological factor in all locations, particularly in the pontine infarcts $(74 \%)$. In posterior infarcts, large arterial atherothrombosis occurred at a higher frequency $(63 \%)$, while cardioembolism was less frequent (12\%). Again, in mesencephalon infarcts and in patients with concomitant infarcts in other locations in addition to brainstem infarcts, cardioembolic etiology was more common ( $26 \%$ and $31.5 \%$, respectively). Dissections involve vertebral arteries in particular. In our patients, dissection-induced infarcts were especially more common in the lateral part of the medulla supplied by Vas.

\section{Conclusion}

Identification of the pathogenetic factors in stroke carries clinical, practical, and scientific significance. Also, determination of risk factors and infarct localization as well as the prediction of etiological parameters may assist in improving survival rates and therapeutic approaches.

Potential limitations of our study include its singlecenter design, small number of certain subpopulations due to large number of study groups, co-existence of risk factors and signs suggestive of more than 1 etiological factor, and inclusion of the same patients in different localization subgroups due to the occurrence of multiple concurrent infarcts.

Further multi-center studies involving larger number of patients will provide deeper insights into this area. 


\section{Disclosure}

The authors report no conflicts of interest in this work.

\section{References}

1. Bamford J, Sandercock P, Dennis M, Burn J, Warlow C. Classification and natural history of clinically identifiable subtypes of cerebral infarction. Lancet. 1991;337(8756):1521-1526.

2. Bogousslavsky J, Regli F, Maeder P, Meuli R, Nader J. The etiology of posterior circulation infarcts: a prospective study using magnetic resonance imaging and magnetic resonance angiography. Neurology. 1993;43(8):1528-1533.

3. Burger KM, Tuhrim S, Naidich TP. Brainstem vascular stroke anatomy. Neuroimag Clin N Am. 2005;15(2):297-324.

4. Bogousslavsky J, Van Melle G, Regli F. The Lausanne Stroke Registry: analysis of 1,000 consecutive patients with first stroke. Stroke. 1998; 19(9):1083-1092.

5. Turney TM, Garraway WM, Whisnant JP. The natural history of hemispheric and brainstem infarction in Rochester, Minnesota. Stroke. 1984;15(5):790-794.

6. Martin PJ, Chang HM, Wityk R, Caplan LR. Midbrain infarction: associations and aetiologies in the New England Medical Center Posterior Circulation Registry. J Neurol Neurosurg Psychiatry. 1998; 64(3):392-395.

7. Lin $Y$, Zhang L, Bao J, et al. Risk factors and etiological subtype analysis of brainstem infarctions. J Neurol Sci. 2014;338(1-2):118-121.

8. Mohr JP, Caplan LR. Vertebrobasilar Disease. In: Stroke: Pathophysiology, Diagnosis and Management. Philadelphia: Elsevier Saunders; 2011:446-484.

9. Amarenco P, Duyckaerts C, Tzourio C, Hénin D, Bousser MG, Hauw JJ. The Prevalence of ulcerated plaques in the aortic arch in patients with stroke. N Engl J Med. 1992;326(4):221-225.

10. Castaigne P, Lhermitte F, Gautier JC, et al. Arterial occlusions in the vertebral-basilar system. A study of 44 patients with post-mortem data. Brain. 1973;96(1):133-154.

11. Fisher CM. Some neuro-ophthalmological observations. J Neurol Neurosurg Psychiatry. 1967;30(5):383-392.

12. Moossy J. Morphology, sites and epidemiology of cerebral atherosclerosis. Res Publ Proc Assos Res Nerv Ment Dis. 1966;41:1-22.

13. Tunick PA, Kronzon I. Protruding atherosclerotic plaque in the aortic arch of patients with systemic embolization: a new findings seen by transesophageal echocardiography. Am Heart J. 1990;120(3):658-660.

14. Caplan LR. Intracranial branch atheromatous disease: a neglected, understudied and underused concept. Neurology. 1989;39(9):1246-1250.

15. Amarenco P, Hauw JJ, Gautier JC. Arterial pathology in cerebellar infarction. Stroke. 1990;21(9):1299-1305.

16. Amarenco P, Hauw JJ. Cerebellar infarction in the territory of the anterior and inferior cerebellar artery: a clinicopathological study of 20 cases. Brain. 1990;113(Pt 1):139-155.

17. Amarenco P, Rosengart A, DeWitt LD, Pessin MS, Caplan LR. Anterior inferior cerebellar artery territory infarcts: Mechanisms and clinical features. Arch Neurol. 1993;50(2):154-161.

18. Fisher CM, Caplan L. Basilar artery branch occlusion: a cause of pontine infarction. Neurology. 1971;21(9):900-905.

19. Murtagh B, Smalling RW. Cardioembolic stroke. Curr Atherosclr Rep. 2006;8(4):310-316.

20. Khoo CW, Lip GYH. Clinical outcomes of acute stroke patients with atrial fibrillation. Expert Rev Neurother. 2009;7:371-374.

21. Ferro JM. Brain embolism-Answers to practical questions. J Neurol. 2003;250(2):139-147.

22. Chiras J, Marciano S, Vega Molina J, Touboul J, Poirier B, Bories J. Spontaneous dissecting aneurysm of the extracranial vertebral artery (20 cases). Neuroradiology. 1985;27(4):327-333.
23. Mas JL, Bousser MG, Hasboun D, Laplane D. Extracranial vertebral artery dissection: a review of 13 cases. Stroke. 1987;18(6):1037-1047.

24. Mokri B, Hauser OW, Sandok BA, Piepgras DG. Spontaneous dissections of the vertebral arteries. Neurology. 1988;38(6):880-885.

25. Kameda W, Kawanami T, Kurita K, et al; Study Group of the Association of Cerebrovascular Disease in Tohoku. Lateral and medial medullary infarction: a comparative analysis of 214 patients. Stroke. 2004; 35(3):694-699.

26. Bassetti C, Bogousslavsky J, Barth A, Regli F. Isolated infarcts of the pons. Neurology. 1996;46(1):165-175.

27. Kumral E, Bayulkem G, Akyol A, Yunten N, Sirin H, Sagduyu A. Mesencephalic and associated posterior circulation infarcts. Stroke. 2002;33(9):2224-2231.

28. Mirsattari SM, McGinn GJ, Halliday WC. Neuro-Behcet disease with predominant involvement of the brainstem. Neurology. 2004;63(2): 382-384.

29. Graus F, Rogers LR, Posner JB. Cerebrovascular complications in patients with cancer. Medicine (Baltimore). 1985;64(1):16-35.

30. Kumral E, Bayulkem G, Evyapan D. Clinical spectrum of pontine infarction. Clinical-MRI correlations. J Neurol. 2002;249(12):1659-1670.

31. Caplan LR, Wityk RJ, Glass TA, et al. New England Medical Center Posterior Circulation registry. Ann Neurol. 2004;56(3):389-398.

32. Vemmos KN, Spengos K, Tsivgoulis G, Manios E, Zis V, Vassilopoulos D. Aetiopathogenesis and long-term outcome of isolated pontine infarcts. J Neurol. 2005;252(2):212-217.

33. Kim K, Lee HS, Jung YH, et al. Mechanism of medullary infarction based on arterial territory involvement. J Clin Neurol. 2012;8(2):116-122.

34. Hosoya T, Adachi M, Yamaguchi K, Haku T, Kayama T, Kato T. Clinical and neuroradiological features of intracranial vertebrobasilar artery dissection. Stroke. 1999;30(5):1083-1090.

35. Seçkin H, Ateş O, Bauer AM, Başkaya MK. Microsurgical anatomy of the posterior spinal artery via a far-lateral transcondylar approach. J Neurosurg Spine. 2009;10(3):228-233.

36. Shono Y, Koga M, Toyoda K, et al. Medial medullary infarction identified by diffusion-weighted magnetic resonance imaging. Cerebrovasc Dis. 2010;30(5):519-524.

37. Amerenco P, Lewy C, Cohen A, Touboul PJ, Roullet E, Bousser MG. Causes and mechanisms of territorial and non territorial cerebellar infarcts in 115 consecutive cases. Stroke. 1994;25(1):105-112.

38. Amarenco P, Hauw JJ. Cerebellar infarction in the territory of the superior cerebellar artery: a clinicopathologic study of 33 cases. Neurology. 1990;40(9):1383-1390.

39. Chaves CJ, Caplan LR, Chung CS, et al. Cerebellar infarcts in the New England Medical Center Posterior Circulation Stroke Registry. Neurology. 1994;44(8):1385-1390.

40. Barth A, Bogousslavsky J, Regli F. The clinical and topographic spectrum of cerebellar infarcts: a clinical-magnetic resonance imaging correlation study. Ann Neurol. 1993;33(5):451-456.

41. Hinshaw DB Jr, Thompson JR, Hasso AN, Casselman ES. Infarction of the brainstem and cerebellum: a correlation of computed tomography and angiography. Radiology. 1980;137(1 Pt 1):105-112.

42. Kase CS, Norrving B, Levine SR, et al. Cerebellar infarction. Clinical and anatomic observations in 66 cases. Stroke. 1993;24(1):76-83.

43. Amarenco P, Hauw JJ, Henin D, et al. [Cerebellar infarction in the area of the posterior cerebellar artery. Clinicopathology of 28 cases]. Rev Neurol. 1989;145(4):277-286. French.

44. Kim JS, Kim J. Pure midbrain infarction: clinical, radiologic, and pathophysiologic findings. Neurology. 2005;64(7):1227-1232. 


\section{Publish your work in this journal}

Neuropsychiatric Disease and Treatment is an international, peerreviewed journal of clinical therapeutics and pharmacology focusing on concise rapid reporting of clinical or pre-clinical studies on a range of neuropsychiatric and neurological disorders. This journal is indexed on PubMed Central, the 'PsycINFO' database and CAS,

and is the official journal of The International Neuropsychiatric Association (INA). The manuscript management system is completely online and includes a very quick and fair peer-review system, which is all easy to use. Visit http://www.dovepress.com/testimonials.php to read real quotes from published authors.

Submit your manuscript here: http://www.dovepress.com/neuropsychiatric-disease-and-treatment-journal 